\title{
Speckle imaging of binary stars: Use of ratios of twofold probability density functions ${ }^{\star}$
}

\author{
M. Carbillet, C. Aime, É. Aristidi, and G. Ricort \\ Département d'Astrophysique de l'Université de Nice-Sophia Antipolis, Unité Mixte de Recherche 6525 du Centre Nationale de \\ la Recherche Scientifique, Parc Valrose, 06108 Nice Cedex 2, France
}

Received November 18, 1996; accepted May 20, 1997

\begin{abstract}
The probability imaging technique applied to double stars speckle data is presented within the framework of a new approach, giving more directly the intensity ratio and relative position of the components. The twofold probability density function is used for this purpose. A theoretical model is developed, pointing out a relevant quantity deduced from the twofold probability density functions of the binary system and a nearby reference star. A method using this quantity is proposed to reconstruct the binary system, together with a reference-less version of it. The practical implementation of the method is tested for limiting cases and is improved by numerical simulations. Making use of the resulting procedure, intensity ratios and relative positions of the components are obtained for three close binary stars: $\beta$ Del, Moaï 1 = SAO 12917 and $\gamma$ Per.
\end{abstract}

Key words: methods: data analysis - techniques: interferometric - binaries: visual — stars: individual: $\beta$ Del — stars: individual: SAO 12917 - stars: individual: $\gamma$ Per

\section{Introduction}

An alternative to the usual computation of moments in the different speckle interferometry techniques (Labeyrie 1970; Knox \& Thompson 1974; Weigelt 1977) is the analysis of the probability density functions (PDFs) at several points in space of the speckle pattern, describing the jointoccurrence of given intensities at several spatial locations.

This original technique - the probability imaging (PI) technique - was firstly proposed by Aime (1987), for binary stars speckle imaging. Extended to the case of a

Send offprint requests to: M. Carbillet

(marcel@procyon.unice.fr)

* Based on observations obtained at Bernard Lyot telescope, Pic du Midi de Bigorre, France, and William Hershell telescope, La Palma, Spain. general object (Aime \& Aristidi 1991; Aime et al. 1993), this technique is turned out to be rather heavy in its application for objects with more than three elementary components. First and foremost because of the number of dimensions of the PDFs that have to be handled (the exhaustive analysis of the specklegrams of a triple star requires a threefold PDF, and so on), but also because there is no simple separation between a function that depends on the object alone and a function that is relevant to the point-source spread speckle pattern in the result, as in the more classical moment analysis. This led us to focus our attention on the binary star problem, for which a twofold PDF analysis is sufficient. These functions are finally proved to be a powerfull and easy-handled tool, giving really interesting results in the domain of relative photometry (Aristidi et al. 1997b).

We present here a new practical implementation of the PI technique well suited for the image reconstruction of binary systems from visible speckle interferometry data. In that sense, this technique is to be compared to other binary-star-oriented techniques, such as the Directed Vector Autocorrelation (Bagnuolo et al. 1992) combined with the fork algorithm, that can be used for binary stars speckle data (Bagnuolo et al. 1990); or to the more recent cross-correlation method proposed by Aristidi et al. (1997a), if coupled to the fork algorithm or to the present $Q$ function computation.

This paper follows the work of Carbillet et al. (1996a) who first obtained - using the PI technique - quantitative results suitable for astrophysical interpretations, from onedimensional near-infrared data. While the previous approach was parametric and made use of minimization techniques, the present one gives the information required from the binary stars data more directly. As in the first application of this PDFs analysis, we find that the major capability of this technique is to give an accurate relative photometry of binary stars. As a matter of fact, this technique seems to be less sensitive to the variations of seeing conditions than the standard speckle techniques. 
The paper is organized as follows. The problem of imaging a binary star by using PDFs is exposed in Sect. 2. A theoretical model of the PDFs that leads to a relevant quantity is exposed in Sect. 3, together with the procedure using it as a tool for speckle imaging. A proposal to get rid of the use of a reference star is described in Sect. 4. Numerical simulations done in order to test the validity and limits of the method are presented in Sect. 5 . An application to real data of the binaries $\beta$ Del, Moaï 1 and $\gamma$ Per is performed in Sect. 6. A discussion of the work (including further planned applications) is given in Sect. 7, and a conclusion in Sect. 8.

\section{Imaging a binary star by using PDFs}

A binary system, for which none of the stars is individually resolved by the telescope, is the most simple object that can be considered for image reconstruction. Its perfect image is made of two points of intensities $I_{1}$ and $I_{2}$, separated by a vector of position $\boldsymbol{d}$ corresponding to the angular separation.

Let us denote as $S(\boldsymbol{r})$ the instantaneous monochromatic speckle pattern produced at the focus of the telescope by a point-source (i.e. a single star unresolved by the telescope, or a reference star). $S(\boldsymbol{r})$ is therefore the point-spread function (PSF) if one considers a unit mean intensity. Assuming isoplanatism, the observed binary star speckle pattern $B(\boldsymbol{r})$ can be written as:

$$
\begin{aligned}
B(\boldsymbol{r}) & =I_{1} S(\boldsymbol{r})+I_{2} S(\boldsymbol{r}-\boldsymbol{d}) \\
& =I_{0}\left(\frac{1}{1+\alpha} S(\boldsymbol{r})+\frac{\alpha}{1+\alpha} S(\boldsymbol{r}-\boldsymbol{d})\right)
\end{aligned}
$$

where: $\alpha=I_{2} / I_{1}$, and $I_{0}$ is the intensity of the binary system corresponding to its overall magnitude.

The relevant information for the imaging of the binary is contained in the three parameters $I_{1}, I_{2}$ and $\boldsymbol{d}$, or equivalently in the three parameters $I_{0}, \alpha$ and $\boldsymbol{d}$. Unless very accurate photometry is performed, we cannot access the absolute value of $I_{0}$, so the imaging parameters to retrieve are $\alpha$ and $\boldsymbol{d}$. Whereas $\boldsymbol{d}$ (or equivalently $-\boldsymbol{d}$ ) and the value (greater or not than 1) of $\alpha$ give a point in the orbit of the binary, an accurate value of $\alpha$ leads to relative photometry of the system. The object of our analysis will be therefore to obtain with no ambiguity $\boldsymbol{d}$ and $\alpha$. Let us now show how an analysis of the PDFs can achieve this goal.

Let us first denote $\Omega_{1}$ the intensity value taken by $I(\boldsymbol{r})$ and $\Omega_{2}$ that of $I(\boldsymbol{r}+\boldsymbol{\rho})$, where $I(\boldsymbol{r})$ describes the intensity distribution in the speckle pattern at a position $\boldsymbol{r}$, and $\boldsymbol{\rho}$ is a space-lag. As we assume stationarity in space, the second-order statistics of $I(\boldsymbol{r})$ are completely defined (Lee $1960)$ by the twofold PDF $P^{(2)}\left(\Omega_{1}, \Omega_{2} ; \boldsymbol{\rho}\right)$.

The quantity $P^{(2)}\left(\Omega_{1}, \Omega_{2} ; \boldsymbol{\rho}\right) \mathrm{d} \Omega_{1} \mathrm{~d} \Omega_{2}$ measures the probability that $I(\boldsymbol{r})$ has an intensity value lying in the elementary interval $\left\{\Omega_{1}, \Omega_{1}+\mathrm{d} \Omega_{1}\right\}$ while $I(\boldsymbol{r}+\boldsymbol{\rho})$, of the same speckle pattern, has an intensity value lying in the interval $\left\{\Omega_{2}, \Omega_{2}+\mathrm{d} \Omega_{2}\right\}$.

As discussed by Aime et al. (1990), there is a strong difference between twofold PDFs of speckle patterns produced by a point-source and a binary star. For a given value of $\boldsymbol{\rho}$, the observed PDFs appear as joint occurrence histograms of the discretized values $\Omega_{1}$ and $\Omega_{2}$, and can be represented as gray-level images. As we shall see in what follows, the twofold PDF of a point-source has an overall symmetrical structure in $\Omega_{1}$ and $\Omega_{2}$ whatever the value of $\boldsymbol{\rho}$. Whereas for $\boldsymbol{\rho}$ close to the star separation vector $\boldsymbol{d}$ of the binary, the corresponding twofold PDF of the double star speckle pattern has an arrow-head shape with a trend towards a direction $\Omega_{2}=\alpha \Omega_{1}$. There is a unique relationship between the shape of the twofold PDF and $\alpha$.

Carbillet et al. (1996a) presented a calibration procedure that uses a parametric approach leading to an estimation of the two parameters $\boldsymbol{d}$ and $\alpha$ from onedimensional near-infrared speckle data. We present here a new approach that is found to give better results for twodimensional visible speckle data. The separation $d$ (modulus of $\boldsymbol{d}$ ) and the position angle PA (with a $180^{\circ}$ quadrant indetermination) need within the present framework to be determined by the by now classical power spectrum analysis and visibility function calculus of Labeyrie's technique. We will now focus on the most accurate way possible of determining $\alpha$ (and the absolute quadrant) by using an analysis of the PDF's slices computed for $\boldsymbol{\rho}=\boldsymbol{d}$ (or equivalently $\boldsymbol{\rho}=-\boldsymbol{d})$.

\section{Theoretical model}

\subsection{General expressions}

Let us first recall the definition of the single-fold characteristic function $(\mathrm{CF}) \Phi_{I}^{(1)}(w)$ of $I(\boldsymbol{r})$, the intensity at the focus of the telescope that can be either the PSF $S(\boldsymbol{r})$ or the binary star speckle pattern $B(\boldsymbol{r}) . \Phi_{I}^{(1)}(w)$ is the complex function of the real variable $w$ defined as:

$\Phi_{I}^{(1)}(w)=E[\exp \{i w I(\boldsymbol{r})\}]=\int \exp \{i w \Omega\} P_{I}^{(1)}(\Omega) \mathrm{d} \Omega$,

where the symbol $E[\bullet]$ denotes the expected value of -, and $P_{I}^{(1)}(\Omega)$ is the single-fold PDF - and the inverse Fourier transform of $\Phi_{I}^{(1)}(w)$.

By generalizing Eq. (2) to two dimensions, we can derive the twofold $\mathrm{CF}$ of $I(\boldsymbol{r})$ :

$\Phi_{I}^{(2)}\left(w_{1}, w_{2} ; \boldsymbol{\rho}\right)=E\left[\exp \left\{i w_{1} I(\boldsymbol{r})+i w_{2} I(\boldsymbol{r}+\boldsymbol{\rho})\right\}\right]$.

On substituting $S(\boldsymbol{r})$ to $I(\boldsymbol{r})$ in the above equation, we directly obtain the twofold CF of the PSF as:

$\Phi_{S}^{(2)}\left(w_{1}, w_{2} ; \boldsymbol{\rho}\right)=E\left[\exp \left\{i w_{1} S(\boldsymbol{r})+i w_{2} S(\boldsymbol{r}+\boldsymbol{\rho})\right\}\right]$ 
while, if $I(\boldsymbol{r})$ represents the binary star speckle pattern $B(\boldsymbol{r})$, Eq. (3) takes the following form:

$$
\begin{aligned}
& \Phi_{B}^{(2)}\left(w_{1}, w_{2} ; \boldsymbol{\rho}\right)=E\left[\exp \left\{i w_{1} B(\boldsymbol{r})+i w_{2} B(\boldsymbol{r}+\boldsymbol{\rho})\right\}\right] \\
& =E\left[\operatorname { e x p } \left\{i w_{1} \frac{1}{1+\alpha} S(\boldsymbol{r})+i w_{1} \frac{\alpha}{1+\alpha} S(\boldsymbol{r}-\boldsymbol{d})\right.\right. \\
& \left.\left.+i w_{2} \frac{1}{1+\alpha} S(\boldsymbol{r}+\boldsymbol{\rho})+i w_{2} \frac{\alpha}{1+\alpha} S(\boldsymbol{r}-\boldsymbol{d}+\boldsymbol{\rho})\right\}\right]
\end{aligned}
$$

In the particular case when $\rho$ is equal to the star separation $\boldsymbol{d}$, this last equation becomes:

$$
\begin{aligned}
& \Phi_{B}^{(2)}\left(w_{1}, w_{2} ; \boldsymbol{\rho}=\boldsymbol{d}\right)= \\
& E\left[\operatorname { e x p } \left\{i \frac{\alpha w_{1}}{1+\alpha} S(\boldsymbol{r}-\boldsymbol{d})+i \frac{w_{2}}{1+\alpha} S(\boldsymbol{r}+\boldsymbol{d})\right.\right. \\
& \left.\left.+i \frac{w_{1}+\alpha w_{2}}{1+\alpha} S(\boldsymbol{r})\right\}\right]
\end{aligned}
$$

As shown by Aime et al. (1993), this expression can be written as a central slice of the threefold $\mathrm{CF}$ of $S(\boldsymbol{r})$. A much simpler expression can be used if we assume that the separation $d$ is large with respect to the speckle size $s$, so that $S(\boldsymbol{r}), S(\boldsymbol{r}-\boldsymbol{d})$ and $S(\boldsymbol{r}+\boldsymbol{d})$ are statistically independent from one another. In that case, assuming that the process is stationary in space, the twofold CF reduces to the product of single-fold CFs of $S(\boldsymbol{r})$ :

$\Phi_{B}^{(2)}\left(w_{1}, w_{2} ; \boldsymbol{\rho}=\boldsymbol{d}\right)=$

$\Phi_{S}^{(1)}\left(\frac{w_{1}+\alpha w_{2}}{1+\alpha}\right) \Phi_{S}^{(1)}\left(\frac{w_{2}}{1+\alpha}\right) \Phi_{S}^{(1)}\left(\frac{\alpha w_{1}}{1+\alpha}\right)$.

By Fourier-inverting this last equation, it leads to (Aime 1993):

$P_{B}^{(2)}\left(\Omega_{1}, \Omega_{2} ; \boldsymbol{\rho}=\boldsymbol{d}\right)=$

$\frac{(1+\alpha)^{3}}{\alpha}\left[P_{S}^{(1)}\left(\frac{1+\alpha}{\alpha} \Omega_{1}\right) P_{S}^{(1)}\left((1+\alpha) \Omega_{2}\right)\right]$

$*\left[P_{S}^{(1)}\left((1+\alpha) \Omega_{1}\right) \delta\left(\alpha \Omega_{1}-\Omega_{2}\right)\right]$,

where $*$ stands for a two-dimensional convolution and $\delta$ is the Dirac distribution.

\subsection{Gaussian model}

We shall now assume that the complex amplitude of the wave at the focus of a large telescope is a circular Gaussian process, i.e. real and imaginary parts of the wave are independent and have identical Gaussian densities. This corresponds to a fully developed speckle pattern. In that case, the intensity of the PSF - that we defined with mean intensity equal to one - follows the well known negative exponential law:

$P_{S}^{(1)}(\Omega)=\exp \{-\Omega\}$

By substituting this last equation into Eq. (8), one obtains the twofold PDF in the normal case (Aime 1993). In the present paper, we shall write this expression as:

$P_{B}^{(2)}\left(\Omega_{1}, \Omega_{2} ; \boldsymbol{\rho}=\boldsymbol{d}\right)=$

$\frac{(1+\alpha)^{3}}{1+\alpha^{3}} \exp \left\{-\left(\Omega_{1}+\Omega_{2}\right)\right\} \exp \left\{-\left(\frac{\Omega_{1}}{\alpha}+\alpha \Omega_{2}\right)\right\}$

$\times\left[\exp \left\{\frac{1+\alpha^{3}}{\alpha} \operatorname{Min}\left(\Omega_{1}, \frac{\Omega_{2}}{\alpha}\right)\right\}-1\right]$,

where we have underscored the term $\exp \left\{-\left(\Omega_{1}+\Omega_{2}\right)\right\}$ that corresponds to the twofold PDF of the PSF, within the assumption of statistical independence used to deduce Eq. (7) from Eq. (6). In that case, the twofold PDF of the binary star appears as the product of the twofold PDF of the PSF and a function denoted as $Q\left(\Omega_{1}, \Omega_{2}\right)$, and defined as the following ratio:

$Q\left(\Omega_{1}, \Omega_{2}\right)=\frac{P_{B}^{(2)}\left(\Omega_{1}, \Omega_{2} ; \boldsymbol{\rho}=\boldsymbol{d}\right)}{P_{S}^{(2)}\left(\Omega_{1}, \Omega_{2} ; \boldsymbol{\rho}\right)}$.

As we shall see in the following, the function $Q\left(\Omega_{1}, \Omega_{2}\right)$ makes it very easy to recover the value of $\alpha$. This is illustrated in Fig. 1 that shows the respective shapes of $P_{B}^{(2)}$, $P_{S}^{(2)}$ and $Q$, computed for the Gaussian model. From these gray-level representations, one can immediately note how the information about $\alpha$, already present in the twofold PDF of the binary, is tremendously enhanced in the $Q$ function.

Let us now describe how the information about $\alpha$ is present in this function. We can write Eq. (11) as:

$$
\begin{aligned}
Q\left(\Omega_{1}, \Omega_{2}\right) & =\frac{(1+\alpha)^{3}}{1+\alpha^{3}} \exp \left\{-\left(\frac{\Omega_{1}}{\alpha}+\alpha \Omega_{2}\right)\right\} \\
& \times\left[\exp \left\{\frac{1+\alpha^{3}}{\alpha} \operatorname{Min}\left(\Omega_{1}, \frac{\Omega_{2}}{\alpha}\right)\right\}-1\right] .
\end{aligned}
$$

The shape of this function is mainly given by the first term inside brackets. The quantity $\operatorname{Min}\left(\Omega_{1}, \frac{\Omega_{2}}{\alpha}\right)$ present in this first exponential divides the $\left(\Omega_{1}, \Omega_{2}\right)$ plane of $Q$ into two regions, with a delimiting ridge of slope $\Omega_{2}=\alpha \Omega_{1}$.

\subsection{Radial integrations}

An easy way to detect the ridge described previously, and shown in Fig. 1 for $\alpha=1.5$, is to radially integrat the $Q$ 

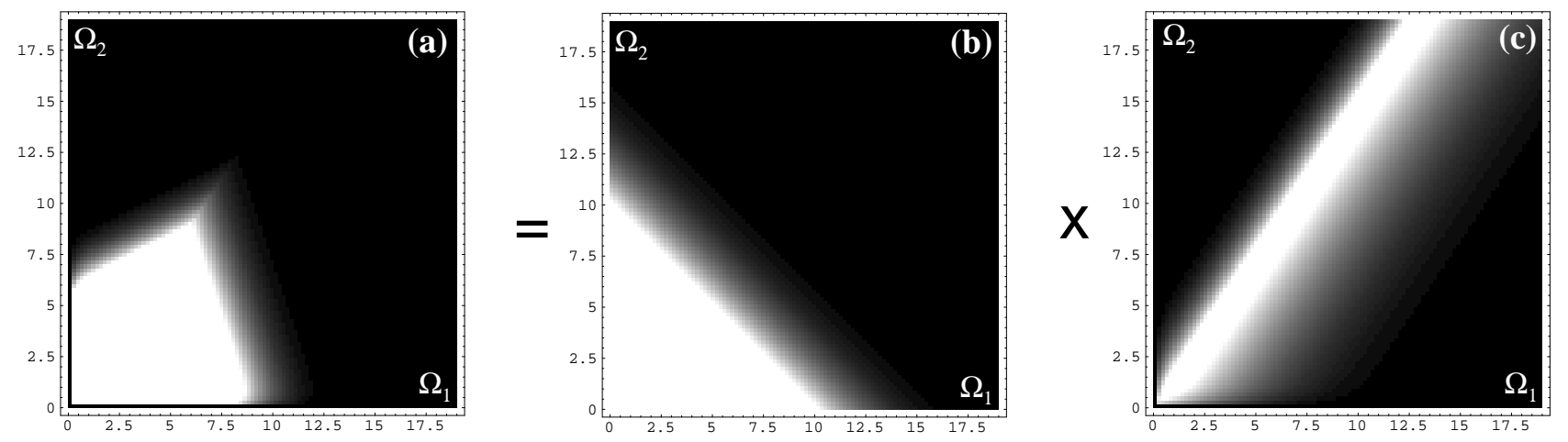

Fig. 1. Gray-level representation of the theoretical twofold PDF of a binary star computed for $\left(\rho_{x}, \rho_{y}\right)=\left(d_{x}, d_{y}\right)$ and $\alpha=1.5$ a), the twofold PDF of a point-source b), and the corresponding $Q$ function c)
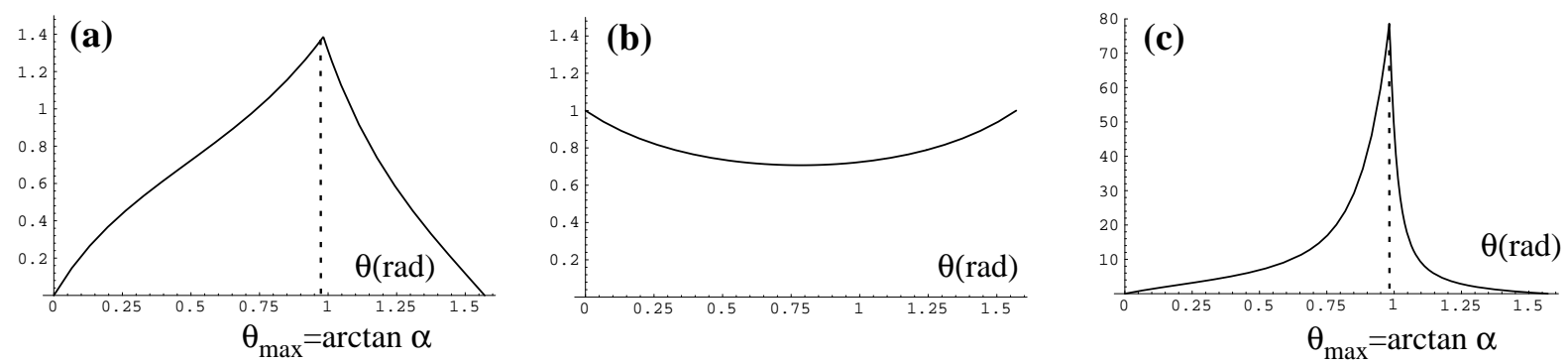

Fig. 2. Plots of the analytical radial integrations for the theoretical twofold PDF of a binary star computed for $\left(\rho_{x}, \rho_{y}\right)=\left(d_{x}, d_{y}\right)$ and $\alpha=1.5 \mathbf{a}$ ), the twofold PDF of a point-source $\mathbf{b}$ ), and the corresponding $Q$ function $\mathbf{c}$ ). These plots precisely correspond to the gray-level representations shown in Fig. 1

function in the $\left(\Omega_{1}, \Omega_{2}\right)$ plane. Analytically, this operation can be written as:

$I_{Q}(\theta)=\int_{0}^{\eta_{\max }} Q(\eta \cos \theta, \eta \sin \theta) \mathrm{d} \eta$

where: $I_{Q}(\theta)$ is the radial integration of $Q, \Omega_{1}=\eta \cos \theta$, $\Omega_{2}=\eta \sin \theta, \eta_{\max }$ is the maximum value of $\eta$, i.e.: $\eta_{\max }=\frac{I_{\max }}{\operatorname{Max}(\cos \theta, \sin \theta)}$ with $I_{\max }$ the actual maximum value of intensity determined by the practical binning. We can first consider the ideal case where $\eta_{\max } \rightarrow \infty$. Then $I_{Q}(\theta)$ becomes:

$$
\begin{aligned}
I_{Q}(\theta) & =\frac{\alpha(1+\alpha)^{3}}{1+\alpha^{3}} \\
& \times\left[\frac{1}{\cos \theta+\alpha^{2} \sin \theta-\left(1+\alpha^{3}\right) \operatorname{Min}\left(\cos \theta, \frac{\sin \theta}{\alpha}\right)}\right. \\
& \left.-\frac{1}{\cos \theta+\alpha^{2} \sin \theta}\right] .
\end{aligned}
$$

Here again, the main part of $I_{Q}(\theta)$ comes from the first term, the second one being almost negligible compared to it. Moreover, the quantity $\operatorname{Min}\left(\cos \theta, \frac{\sin \theta}{\alpha}\right)$ divides the axis of $\theta$ into two regions, causing the relevant behavior:

$$
I_{Q}(\theta) \rightarrow \infty \text { for } \theta=\arctan \alpha \text {. }
$$

This is due to the fact that $Q\left(\Omega_{1}, \Omega_{2}\right)$ rapidly converges to $\frac{(1+\alpha)^{3}}{1+\alpha^{3}}$ for $\Omega_{2}=\alpha \Omega_{1}$ (that corresponds to $\theta=\arctan \alpha$ ), and as $\Omega_{2}$ increases. This general behavior will allow us easily to find the exact value of $\alpha$ by searching for the infinite maximum of $I_{Q}(\theta)$.

In practice, we have to consider that $\eta_{\max }$ has a finite value. In that case the value of $I_{Q}(\theta)$ becomes finite too, but the main figure is kept: $I_{Q}(\theta)$ has a very clear maximum for the right value $\alpha=\tan \theta$ of the intensity ratio of the binary star. Figure 2 shows $I_{Q}(\theta)$ compared to the radial integrations performed on the twofold PDF of the PSF and on the twofold PDF of a binary star speckle pattern. As one can see from these plots, the maximum of both the radial integrations of $P_{B}^{(2)}$ and $Q$ gives the value of $\alpha$, but the maximum of $Q$ is $\sim 30$ times higher (for the present case where $I_{\max }=19$ ) and much better defined.

\section{Avoiding the use of a reference star}

The use of a reference star is generally needed in speckle interferometry to correct the quantity computed from atmospheric effects. We saw for instance in the previous sections that we derive $Q$ computing the ratio of the twofold PDF of the binary star and the twofold PDF of the PSF (even if in this case this does not exactly 

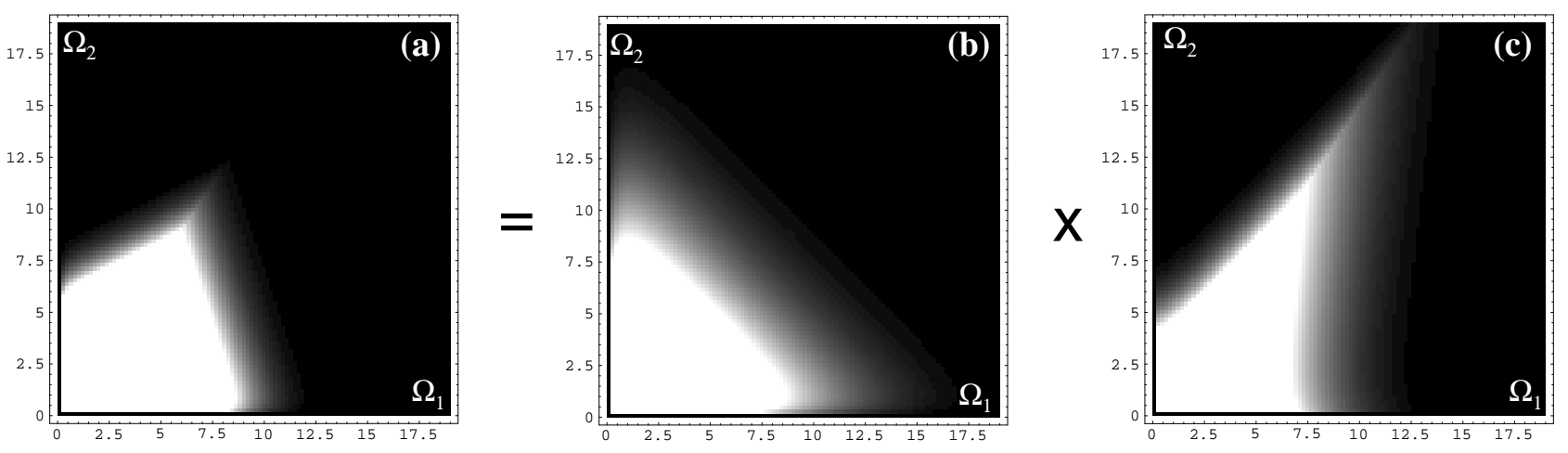

Fig. 3. Top: gray-level representation of the theoretical twofold PDF of a binary star computed for $\left(\rho_{x}, \rho_{y}\right)=\left(d_{x}, d_{y}\right)$ a), the twofold PDF of same binary computed for $\left.\left(\rho_{x}, \rho_{y}\right) \perp\left(d_{x}, d_{y}\right) \mathbf{b}\right)$, and the corresponding $\tilde{Q}$ function $\left.\mathbf{c}\right)$

correspond to a complete correction of the atmospheric effects). Nevertheless, since seeing conditions can rapidly change (Coulman 1985), the twofold PDF of the PSF can be badly estimated from the observation of a reference star. In that case, it can be useful to avoid the use of the reference star data. This can be done by using the present technique.

We have considered so far the twofold PDF of a binary star just for the space-lag vector $\boldsymbol{\rho}$ equal to the separation vector $\boldsymbol{d}$, i.e. when the information about the binarity of the object is maximum. Let us now consider the inverse case, i.e. the case for which $\Omega_{1}$ and $\Omega_{2}$ are uncorrelated. Within the model assumed here, this occurs whenever $\boldsymbol{\rho} \neq$ $\boldsymbol{d}$ and $\rho \neq 0$. In practice, and considering the effects due to the real extension of the speckle pattern, we chose to consider the particular vector $\boldsymbol{\rho} \perp \boldsymbol{d}$ (with the length $\rho=$ $d$ ), for which on the one hand $\Omega_{1}$ and $\Omega_{2}$ are supposed to be uncorrelated, and on the other the effects due to the low frequencies present in the speckle pattern are supposed to be similar.

Considering again Eq. (5), we have $S(\boldsymbol{r}), S(\boldsymbol{r}-\boldsymbol{d})$, $S(\boldsymbol{r}+\boldsymbol{\rho})$ and $S(\boldsymbol{r}-\boldsymbol{d}+\boldsymbol{\rho})$ that are still statistically independent from one another, all the more so because $d, \rho$ and $|\boldsymbol{\rho}-\boldsymbol{d}|$ are large in comparison to $s$. Assuming again that the process is stationary in space, we can write:

$$
\begin{aligned}
\Phi_{B}^{(2)}\left(w_{1}, w_{2} ; \boldsymbol{\rho} \perp \boldsymbol{d}\right) & =\Phi_{S}^{(1)}\left(\frac{w_{1}}{1+\alpha}\right) \Phi_{S}^{(1)}\left(\frac{\alpha w_{1}}{1+\alpha}\right) \\
& \times \Phi_{S}^{(1)}\left(\frac{w_{2}}{1+\alpha}\right) \Phi_{S}^{(1)}\left(\frac{\alpha w_{2}}{1+\alpha}\right)
\end{aligned}
$$

Taking the same kind of assumption as in Sect. 3 and following the same process, leads to, if $\alpha \neq 1$ :

$$
\begin{aligned}
P_{B}^{(2)}\left(\Omega_{1}, \Omega_{2} ; \boldsymbol{\rho} \perp \boldsymbol{d}\right)= & \frac{(1+\alpha)^{2}}{(1-\alpha)^{2}}\left[\exp \left\{-(1+\alpha)\left(\Omega_{1}+\Omega_{2}\right)\right\}\right. \\
& -\exp \left\{-(1+\alpha)\left(\Omega_{1}+\frac{\Omega_{2}}{\alpha}\right)\right\}
\end{aligned}
$$

$$
\begin{aligned}
& -\exp \left\{-(1+\alpha)\left(\frac{\Omega_{1}}{\alpha}+\Omega_{2}\right)\right\} \\
& \left.+\exp \left\{-\frac{1+\alpha}{\alpha}\left(\Omega_{1}+\Omega_{2}\right)\right\}\right]
\end{aligned}
$$

and, if $\alpha=1$ :

$$
P_{B}^{(2)}\left(\Omega_{1}, \Omega_{2} ; \boldsymbol{\rho} \perp \boldsymbol{d}\right)=16 \Omega_{1} \Omega_{2} \exp \left\{-2\left(\Omega_{1}+\Omega_{2}\right)\right\} .
$$

As in Sect. 3 one deduces from these quantities that:

$P_{B}^{(2)}\left(\Omega_{1}, \Omega_{2} ; \boldsymbol{\rho} \perp \boldsymbol{d}\right)=P_{S}^{(2)}\left(\Omega_{1}, \Omega_{2} ; \boldsymbol{\rho}\right) U\left(\Omega_{1}, \Omega_{2}\right)$,

where, if $\alpha \neq 1$ :

$$
\begin{aligned}
U\left(\Omega_{1}, \Omega_{2}\right)= & \frac{(1+\alpha)^{2}}{(1-\alpha)^{2}}\left[\exp \left\{-\frac{1}{\alpha}\left(\Omega_{1}+\Omega_{2}\right)\right\}\right. \\
& -\exp \left\{-\left(\frac{\Omega_{1}}{\alpha}+\alpha \Omega_{2}\right)\right\} \\
& -\exp \left\{-\left(\alpha \Omega_{1}+\frac{\Omega_{2}}{\alpha}\right)\right\} \\
& \left.+\exp \left\{-\alpha\left(\Omega_{1}+\Omega_{2}\right)\right\}\right]
\end{aligned}
$$

and, if $\alpha=1$ :

$$
U\left(\Omega_{1}, \Omega_{2}\right)=16 \Omega_{1} \Omega_{2} \exp \left\{-\left(\Omega_{1}+\Omega_{2}\right)\right\} .
$$

From Eq. (19) and Eq. (11), we can deduce a relationship between the twofold PDF of a binary star computed for $\boldsymbol{\rho}=\boldsymbol{d}$ and computed for $\boldsymbol{\rho} \perp \boldsymbol{d}$ :

$P_{B}^{(2)}\left(\Omega_{1}, \Omega_{2} ; \boldsymbol{\rho}=\boldsymbol{d}\right)=P_{B}^{(2)}\left(\Omega_{1}, \Omega_{2} ; \boldsymbol{\rho} \perp \boldsymbol{d}\right) \tilde{Q}\left(\Omega_{1}, \Omega_{2}\right)$,

where : $\tilde{Q}\left(\Omega_{1}, \Omega_{2}\right)=Q\left(\Omega_{1}, \Omega_{2}\right) / U\left(\Omega_{1}, \Omega_{2}\right)$.

This last relationship is valid as long as $U\left(\Omega_{1}, \Omega_{2}\right)$ is not zero, i.e. for $\Omega_{1} \neq 0$ and $\Omega_{2} \neq 0$ (if $\alpha \neq 1$ ). Let us now show that $\tilde{Q}$ has the same kind of behavior and interest as $Q$. Figure 3 illustrates the relationship given in Eq. (22), 
like in Fig. 1. The twofold PDF of the binary star speckle pattern computed for $\boldsymbol{\rho} \perp \boldsymbol{d}$ appears very similar to the twofold PDF of the PSF, and the above defined $\tilde{Q}$ clearly shows the same kind of form as $Q$. The result is a little less impressive than in Fig. 1, but applications to simulated and real data can lead to an equivalent result. As we shall confirm in what follows, the method suggested in this paper can be used with or without reference star to correct for atmospheric effects (the two versions of the method will be called from now the standard version and the reference-less version). In the next section we shall among other test the validity of this statement by doing some numerical simulations.

\section{Numerical simulations}

We assumed in writing the equations in the previous sections that $d \gg s$, i.e. the separation between the components $d$ is large with respect to the speckle size $s$. In practice, the interesting point for observations is when $d \gtrsim s$, since the aim of every speckle imaging technique is to reach as close as possible the diffraction-limited resolution of the telescope. Then, in order to complete the theoretical study and test the validity and limits of it for practical speckle observations, we chose to make several numerical simulations with different values of $d$ and $\alpha$. We decided not to report all these simulations in this paper but just the most interesting ones, i.e. for a separation $d=\frac{5}{3} s$, and for three different relevant values of $\alpha$.

\subsection{Practical implementation of the method}

The functions $Q$ and $\tilde{Q}$ are respectively obtained by dividing the twofold PDF of the binary star computed for $\boldsymbol{\rho}=\boldsymbol{d}$ by that of a point-source, and by that of the binary computed for $\boldsymbol{\rho} \perp \boldsymbol{d}$. In order to avoid zero divisions during this operation, we made use of an iterating algorithm based on Van Cittert (1931) and already applied to speckle data by Cruzalèbes et al. (1996). The output estimate of this algorithm perfectly converges to the solution of the normal division after an infinite number of iterations. Let be $A=\frac{B}{C}$. If $|C| \ll 1$ the calculation of $A$ may rapidly diverge. One estimate of $A$ can then be:

$$
A_{n}=B \sum_{i=0}^{n}(1-C)^{i} \text {. }
$$

Because $\sum_{i=0}^{\infty}(1-C)^{i}=\frac{1}{C}$, it is easy to demonstrate that $\lim _{n \rightarrow \infty} A_{n}=A$. In the case where the denominator $C$ becomes smaller than the limit for which the machine cannot see the difference between 1 and $1+C$, we found that it is typically sufficient to perform about ten iterations to estimate the ratio. In the other case, we simply calculated the ratio by normal division.

We also computed the quantity $Q-Q^{T}$ to enhance the relevant ridge, where $Q^{T}$ is the transpose quantity of $Q$.
The radial integration of this quantity $I_{Q-Q^{\mathrm{T}}}$ is related to $I_{Q}$ by:

$$
\begin{aligned}
I_{Q-Q^{\mathrm{T}}} & =\int_{0}^{\eta_{\max }}\left[Q-Q^{\mathrm{T}}\right](\eta \cos \theta, \eta \sin \theta) \mathrm{d} \eta \\
& =I_{Q}(\theta)-I_{Q^{\mathrm{T}}}(\theta)=I_{Q}(\theta)-I_{Q}\left(\frac{\pi}{2}-\theta\right)
\end{aligned}
$$

So its ideal expression (i.e. when $\eta_{\max } \rightarrow \infty$ ) follows:

$$
\left\{\begin{array}{l}
I_{Q-Q^{\mathrm{T}}}(\theta) \rightarrow \infty \quad \text { if } \theta=\arctan \alpha . \\
I_{Q-Q^{\mathrm{T}}}(\theta) \rightarrow-\infty \text { if } \theta=\arctan \frac{1}{\alpha}=\frac{\pi}{2}-\arctan \alpha .
\end{array}\right.
$$

In the present case $\eta_{\max }$ is obviously finite and the general behavior of $I_{Q-Q^{\mathrm{T}}}(\theta)$ is to have a maximum for the right value of $\alpha$, like $I_{Q}(\theta)$, but a minimum too for $\frac{1}{\alpha}$. For such a quantity the extrema are better defined. A second interest is that it could stand out better between a value of $\alpha$ close to but greater than 1 and a value of $\alpha$ close to but smaller than 1 .

In practice, to estimate $Q^{\mathrm{T}}$, we did not directly make use of $Q$. We computed $\left[\left(P_{B}^{(2)}\right)^{\mathrm{T}} / P_{S}^{(2)}\right]$ in order to have two different estimates (as $P_{S}^{(2)}$ is determined experimentally) of the intensity ratio when analyzing the quantity $Q-Q^{\mathrm{T}}$ : one corresponding to the maximum of the radial integration, and one to the minimum. The output values of $\theta$ are then averaged from these two estimates, together with the corresponding errors.

In addition, and in order to get rid of the effect of statistical fluctuations and to keep only the most significant features, we also smoothed the $Q, \tilde{Q}, Q-Q^{\mathrm{T}}$ and $\tilde{Q}-\tilde{Q}^{\mathrm{T}}$ estimates by convolving them by a $3 \times 3$ unit valued filter.

We consider only the part of the computed quantities $Q, \tilde{Q}, Q-Q^{\mathrm{T}}$ and $\tilde{Q}-\tilde{Q}^{\mathrm{T}}$ where the signal-to-noise ratio is the best, i.e. where there is a significant number of events in the twofold PDFs of the reference and the binary star. This typically corresponds, in our present case, to an extraction of $32 \times 32$ pixels near the origin for PDFs computed with a sample of the intensity of 256 levels.

\subsection{General case}

The simulation work presented in this subsection made use of two data sets (one for the binary star and one for the point-source), each made of 100 speckle frames of $128 \times$ 128 pixels, simulated with the following parameters:

- observing wavelength: $\lambda=6500 \AA$,

- Fried's parameter: $r_{0}=20 \mathrm{~cm}$,

- telescope diameter: $D=2 \mathrm{~m}$,

- speckle size: $s=3$ pixels,

- separation vector for the binary: $\boldsymbol{d}=(+3,+4)$ pixels $\Rightarrow d=5$ pixels,

- intensity ratio between the components: $\alpha=1.5$. 

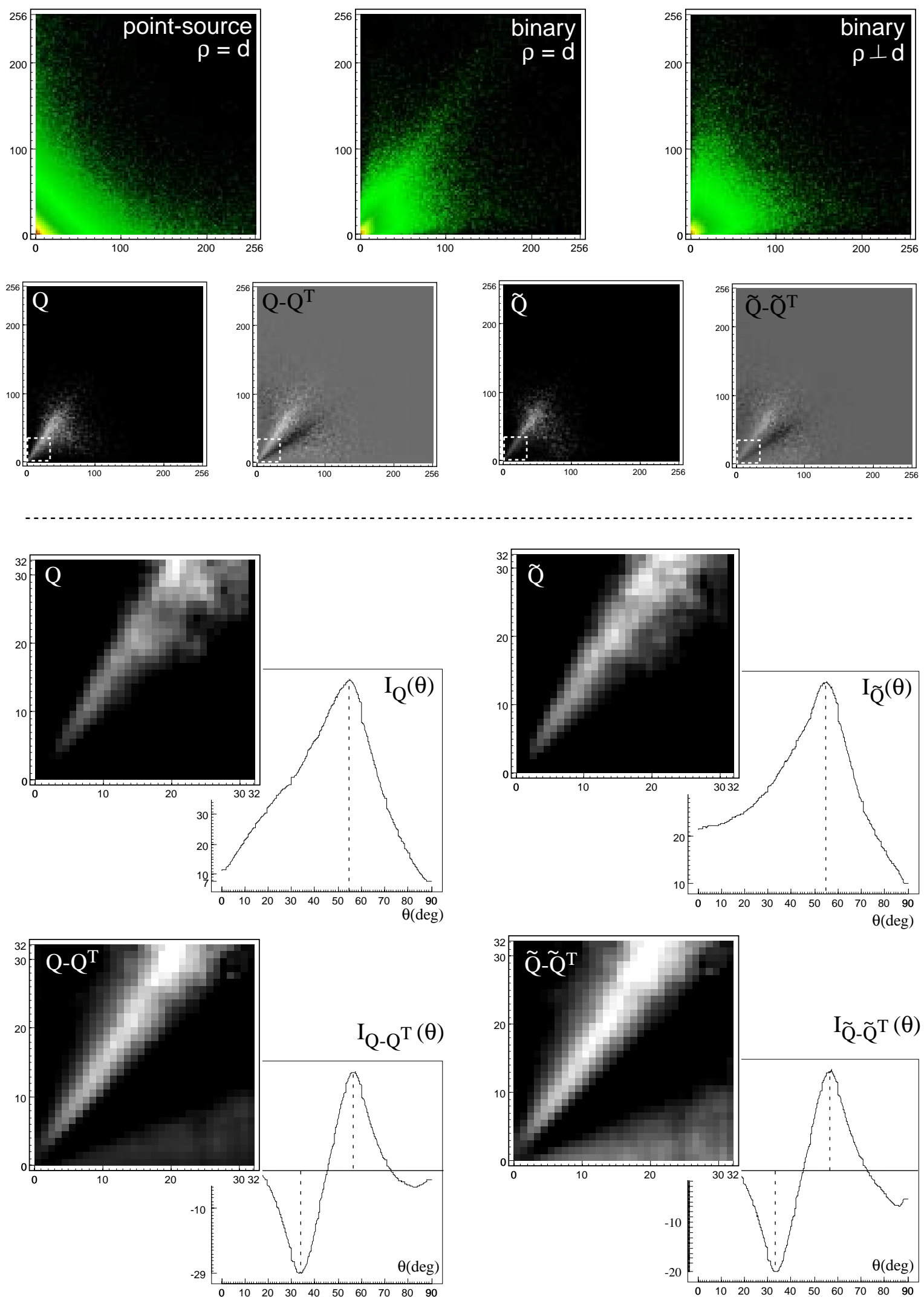

Fig. 4. Top, first row: logarithmic gray-level representation of the twofold PDFs computed for the simulation made for $\alpha=1.5$ and $\left(d_{x}, d_{y}\right)=(+3,+4)$. Top, second row: linear gray-level representation of the corresponding $Q, Q-Q^{\mathrm{T}}, \tilde{Q}$ and $\tilde{Q}-\tilde{Q}^{\mathrm{T}}$ functions. The white squares show the $32 \times 32$ extraction zone used for the computation of the radial integrations. Bottom: extraction of $Q, \tilde{Q}, Q-Q^{\mathrm{T}}$ and $\tilde{Q}-\tilde{Q}^{\mathrm{T}}$, together with their respective radial integrations. Its extrema are outlined by dashed lines 
In Fig. 4 we represent the PDFs obtained for the pointsource for $\boldsymbol{\rho}=\boldsymbol{d}$, and for the binary for $\boldsymbol{\rho}=\boldsymbol{d}$ and for $\boldsymbol{\rho} \perp \boldsymbol{d}$. The functions $Q$ and $\tilde{Q}$ are deduced from this, and represented together with their radial integrations. The functions $Q-Q^{\mathrm{T}}$ and $\tilde{Q}-\tilde{Q}^{\mathrm{T}}$ are represented as well with their radial integrations.

The procedure to quantitatively find the extrema of these radial integrations and to estimate the respective errors makes use of a polynomial fit of these quantities around the extrema. We chose for this purpose to fit the $\pm 2.5^{\circ}$ region surrounding the extrema by a polynomial of the second degree, since close to its maximum a convex function is supposed to have a quadratic-like behavior.

The values of $\theta=\arctan \alpha$ found from the four quantities $I_{Q}(\theta), I_{\tilde{Q}}(\theta), I_{Q-Q^{\mathrm{T}}}(\theta)$ and $I_{\tilde{Q}-\tilde{Q}^{\mathrm{T}}}(\theta)$ are reported in the first row of Table1. The values found for $Q$ and $\tilde{Q}$ are rather less than the input value of $\theta$. This means that there is a systematic error in detecting the right value of $\alpha$ from the maximum of $I_{Q}$ and $I_{\tilde{Q}}$. However this systematic error is not present, or at least in a very small way, in the case, not presented in this paper, where the separation $d$ is actually large with respect to $s$. Nevertheless, this systematic error is avoided by considering the values of $\theta$ found for $Q-Q^{\mathrm{T}}$ and $\tilde{Q}-\tilde{Q}^{\mathrm{T}}$. So while $Q$ or $\tilde{Q}$ gives us a first (but biased) estimate of $\theta$, the computation of $Q-Q^{\mathrm{T}}$ or $\tilde{Q}-\tilde{Q}^{\mathrm{T}}$ then gives us a good value of it. The general procedure will be to consider directly $Q-Q^{\mathrm{T}}$ or $\tilde{Q}-\tilde{Q}^{\mathrm{T}}$ to estimate the intensity ratio of a binary star. Finally, this method gives equivalent results by using it in its reference-less version or in its standard version.

We made several numerical simulations in order to test the validity and limits of the method. This showed us that two kind of limiting cases exist depending on $\alpha$.

\subsection{Limiting cases}

In order to better test the limiting cases of the method, we chose to make the simulation with a larger number of frames per set: 1000. The first limiting case is when $\alpha$ is large (or small) with respect to 1 . This corresponds to a large magnitude difference between the components of the binary star. This is already a well-known limit of speckle observations but in our present case, this corresponds to a ridge of the $Q$ function close to the axis $\Omega_{2}$ (or $\Omega_{1}$ ), implying then a difficult determination of the right value of $\alpha$. We found, with the typical parameters taken here, that the useful limit of the method is for $\alpha \sim 10$ (and for $\alpha \sim 0.1$ ), i.e. for a magnitude difference of $\sim 2.5$. As shown in Table 1 , second row, the computations $Q$ and $\tilde{Q}$ could only give an idea of $\theta$, and we deduce from $I_{Q}$ and $I_{\tilde{Q}}$ that $\theta$ is greater than or of the order of $80^{\circ}$. The estimate of $\theta$ is still available from $I_{Q-Q^{\mathrm{T}}}$ and $I_{\tilde{Q}-\tilde{Q}^{\mathrm{T}}}$ but gives a slight under-estimate.

The second limiting case is when $\alpha$ is close to 1 . This problem occurs when the two components of a binary system are of close magnitudes, implying then an ambiguity in the determination of the PA. In that case, the quantities $Q-Q^{\mathrm{T}}$ and $\tilde{Q}-\tilde{Q}^{\mathrm{T}}$ become very small but still contain the information about the orientation of the binary, even if the precise determination of $\alpha$ is no longer possible. Nevertheless, $Q$ and $\tilde{Q}$ can in this case give a good estimate of it, as shown in Table 1, third row, where we report the result of a simulation made for $\alpha=1.01$ (i.e. for a magnitude difference of $\sim 0.01$ ).

Figure 5 illustrates the procedure used to analyze these two limiting cases. In the first case $(\alpha=10), \theta$ is directly determined from the extrema of $I_{Q-Q^{\mathrm{T}}}$ or $I_{\tilde{Q}-\tilde{Q}^{\mathrm{T}}}$. In the second $(\alpha=1.01), \theta$ is determined from $I_{Q}$ and $I_{\tilde{Q}}$ and the orientation is checked from $I_{Q-Q^{\mathrm{T}}}$ or $I_{\tilde{Q}-\tilde{Q}^{\mathrm{T}}}$. An interesting case is presented by the reference-less version of the method. In fact, while $I_{\tilde{Q}}$ shows a maximum for $\theta$ slightly smaller than $45^{\circ}$ (but with an error large enough to include the value $45^{\circ}$ ), the shape of $I_{\tilde{Q}-\tilde{Q}^{\mathrm{T}}}$ clearly denotes a value of $\theta$ greater than $45^{\circ}$. In conclusion, a good estimate of $\alpha$ can be found by using our method if the following procedure is performed:

1. Compute $Q$ and $\tilde{Q}$.

2. If $\alpha$ is not close to 1: estimate it with $I_{Q-Q^{\mathrm{T}}}$ or $I_{\tilde{Q}-\tilde{Q}^{\mathrm{T}}}$.

3. If $\alpha$ is close to 1: estimate it with $I_{Q}$ or $I_{\tilde{Q}}$ and check the orientation (i.e. check if $\theta$ is greater or not than $45^{\circ}$ ) by using $I_{Q-Q^{\mathrm{T}}}$ or $I_{\tilde{Q}-\tilde{Q}^{\mathrm{T}}}$.

An application of this procedure to real data of close visual binary stars is performed in the next section, together with a comparison with the results found elsewhere.

\section{Application to real data}

Preliminary results obtained with the technique in its standard version were already presented elsewhere (Carbillet et al. 1996b). We give here a more accurate application of the technique in its two versions to three binary stars for which the observing conditions are reported in Table 2. All the data reduced in this section consist of high-light level speckle frames of $128 \times 128$ pixels. We give in what follows a detailed description of the analysis for each object.

\section{1. $\beta$ Del}

The subgiant $\beta$ Del is a close binary of 26.6 years of period given as a standard star for binary-star interferometry by McAlister \& Hartkopf (1983). The latest orbit is computed by Hartkopf et al. (1989). The reference star observed was $\epsilon$ Del from which we used 410 frames, and 324 for the binary.

The separation angle $d$ and the PA from which we derived an estimation of $\boldsymbol{d}$ were computed from the classical calculation of the visibility function, founding: $d=0^{\prime \prime} 22$ and $\mathrm{PA}=288^{\circ} / 108^{\circ}$.

Figure 6 , first row, shows both the quantities $Q-Q^{\mathrm{T}}$ and $\tilde{Q}-\tilde{Q}^{\mathrm{T}}$, with their radial integrations $I_{Q-Q^{\mathrm{T}}}$ and $I_{\tilde{Q}-\tilde{Q}^{\mathrm{T}}}$, derived from the twofold PDFs of the binary and 

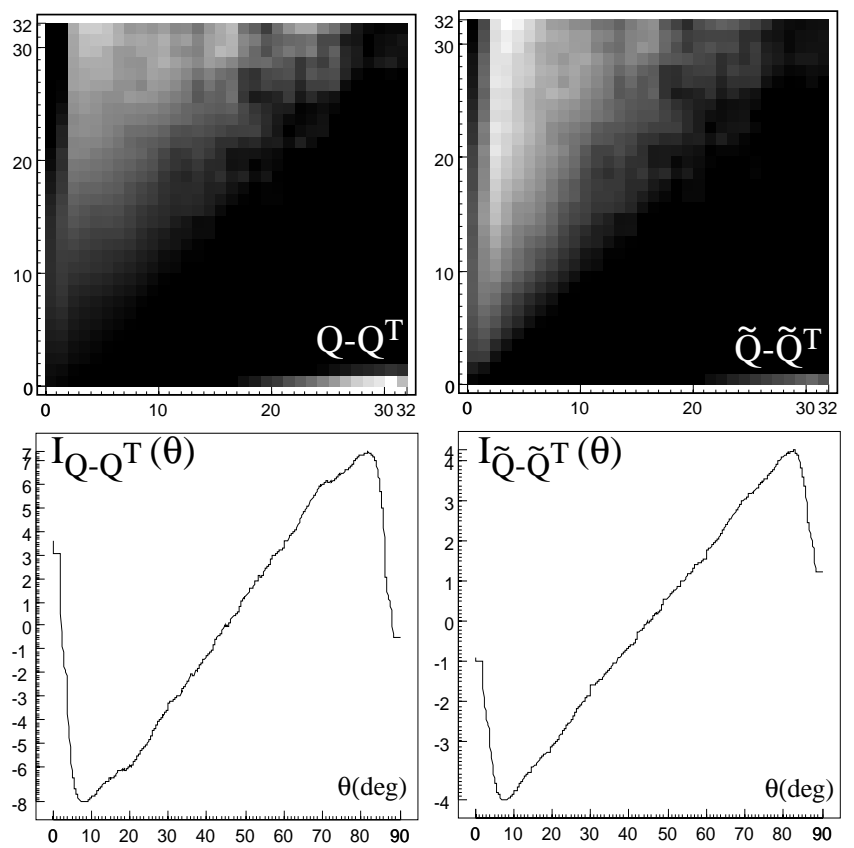
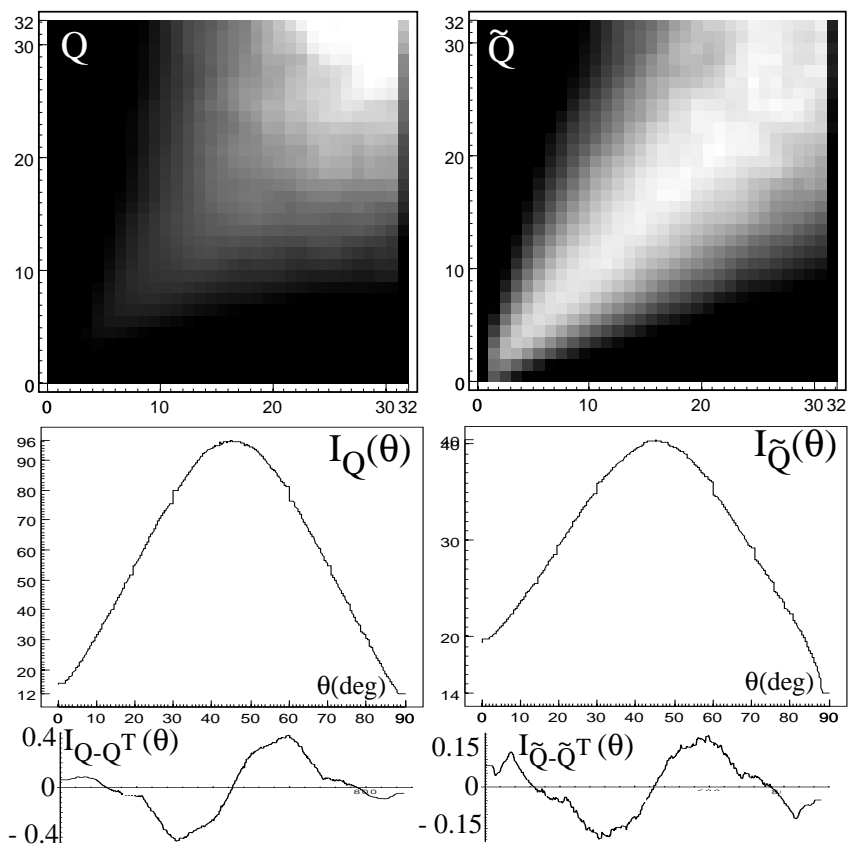

Fig. 5. Left: linear gray-level representation of $Q-Q^{\mathrm{T}}$ and $\tilde{Q}-\tilde{Q}^{\mathrm{T}}$ for $\alpha=10$, together with the plots of $I_{Q-Q^{\mathrm{T}}}(\theta)$ and $I_{\tilde{Q}-\tilde{Q}^{\mathrm{T}}}(\theta)$. Right: linear gray-level representation of $\tilde{Q}$ and $\tilde{Q}-\tilde{Q}^{\mathrm{T}}$ for $\alpha=1.01$, together with the plots of $I_{Q}(\theta), I_{Q-Q^{\mathrm{T}}}(\theta)$, $I_{\tilde{Q}}(\theta)$ and $I_{\tilde{Q}-\tilde{Q}^{\mathrm{T}}}(\theta)$

Table 1. Values of $\theta$ (and corresponding intrinsic errors $\Delta \theta$ ) found for the numerical simulations

\begin{tabular}{|c|c||c|c||c|c|}
\hline input $\alpha$ & input $\theta$ & $Q$ & $Q-Q^{T}$ & $\tilde{Q}$ & $\tilde{Q}-\tilde{Q}^{T}$ \\
\hline \hline 1.5 & 56.31 & $54.85 \pm 0.60$ & $56.40 \pm 0.50$ & $54.80 \pm 0.60$ & $56.35 \pm 0.50$ \\
\hline \hline 10 & 84.29 & $\gtrsim 80$ & $83.80 \pm 0.70$ & $\gtrsim 80$ & $83.70 \pm 0.60$ \\
\hline 1.01 & 45.28 & $45.25 \pm 0.95$ & $>45$ & $44.90 \pm 0.80$ & $>45$ \\
\hline
\end{tabular}

Table 2. Observation table of the three sets of data processed in Sect. 6. The right ascension, the declination and the combined magnitude in the red $\left(m_{R}\right)$ are given, together with the telescope used (wHT = William Hershell Telescope, La Palma, SpainBLT $=$ Bernard Lyot Telescope, Pic du Midi de Bigorre, France), the observing wavelength /bandwidth in $\AA$, the approximate average value of the Fried's parameter $r_{0}$, the exposure time and the date of observation

\begin{tabular}{|c|c|c|c||c|c|c|c|c|}
\hline star name & r.a. 2000.0 & dec. 2000.0 & $m_{R}$ & Telescope & $\lambda / \Delta \lambda$ & $<r_{0}>$ & $\Delta t$ & Date \\
\hline \hline$\beta$ Del & $20^{\mathrm{h}} 37^{\prime} 30^{\prime \prime}$ & $14^{\circ} 36^{\prime} 00^{\prime \prime}$ & 3.2 & $2 \mathrm{~m}$ BLT & $6580 / 425$ & $\sim 20 \mathrm{~cm}$ & $20 \mathrm{~ms}$ & $11 / 09 / 94$ \\
\hline Moaï 1 & $03^{\mathrm{h}} 49^{\prime} 36^{\prime \prime}$ & $63^{\circ} 17^{\prime} 52^{\prime \prime}$ & 6 & $2 \mathrm{~m}$ BLT & $6500 / 700$ & $\sim 30 \mathrm{~cm}$ & $20 \mathrm{~ms}$ & $12 / 12 / 95$ \\
\hline$\gamma$ Per & $03^{\mathrm{h}} 04^{\prime} 48^{\prime \prime}$ & $53^{\circ} 30^{\prime} 24^{\prime \prime}$ & 2.3 & $4.2 \mathrm{~m}$ WHT & $6580 / 425$ & $\sim 20 \mathrm{~cm}$ & $25 \mathrm{~ms}$ & $19 / 01 / 95$ \\
\hline
\end{tabular}

of the reference star computed for the space-lag vector $\boldsymbol{\rho}=\boldsymbol{d}$, and from the twofold PDF of the binary computed for $\boldsymbol{\rho} \perp \boldsymbol{d}$. From these quantities, we could deduce the value of $\theta$ for which they are extrema by the procedure described in Sect. 5. So we have: $\arctan \alpha=(23.85 \pm 0.95)^{\circ}$ using the standard version; or: $\arctan \alpha^{\prime}=(24.5 \pm 1.5)^{\circ}$ using the reference-less version. The exact value of the intensity ratio, giving then both the orientation and a relative photometry of the binary system, is found to be $\alpha=0.440 \pm 0.020$ (or $\alpha^{\prime}=0.455 \pm 0.030$ ), that corresponds to a magnitude difference $\Delta m=0.885 \pm 0.050$ (or $\left.\Delta m^{\prime}=0.855 \pm 0.070\right)$. This is in agreement with the value given by Couteau (1962) - i.e. 0.9 -if one considers that $\Delta m_{V}$ is roughly similar to $\Delta m_{R}$ for this object. Moreover, since we found an intensity ratio smaller than 1 , we can assume, given the orientation of the frames and $\rho$, that PA is $288^{\circ}$ and not $108^{\circ}$. This is anyway what was expected from the orbit cited before. 

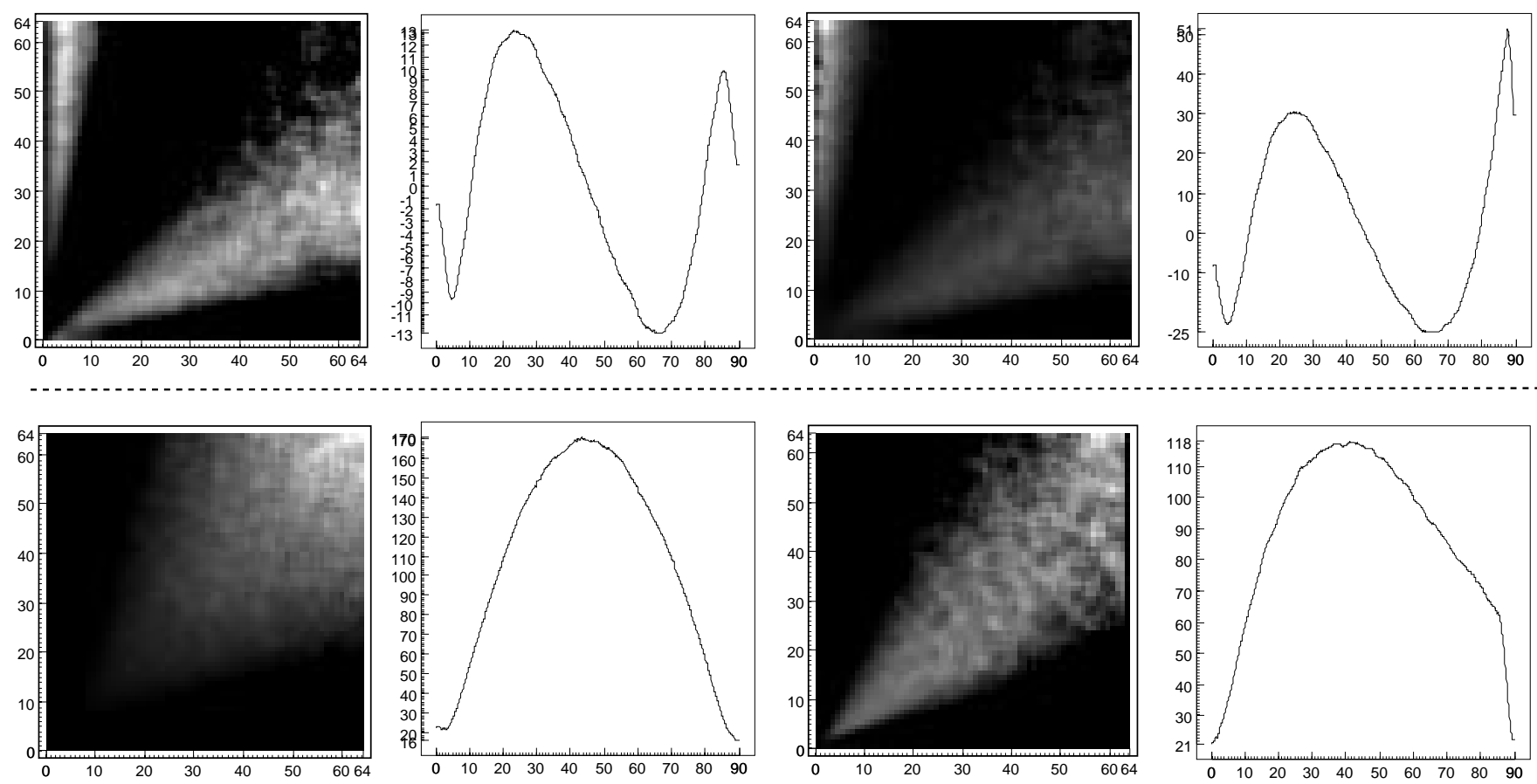

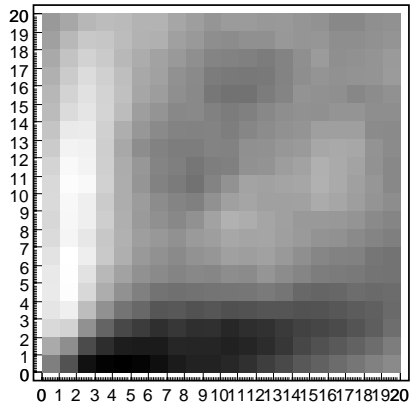

(a)

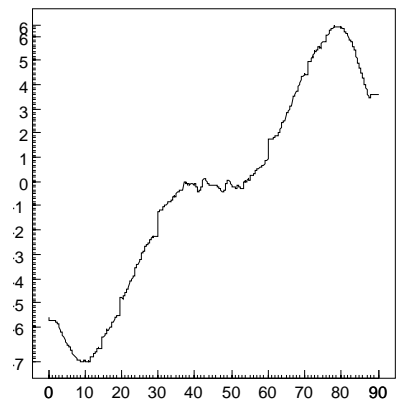

(b)

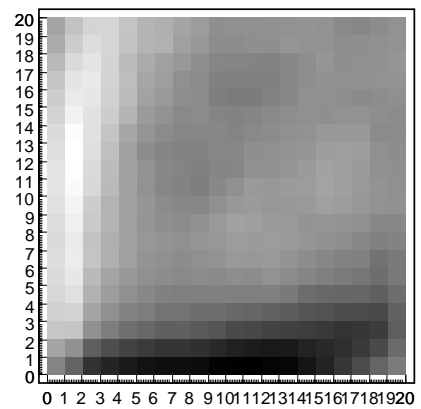

(c)

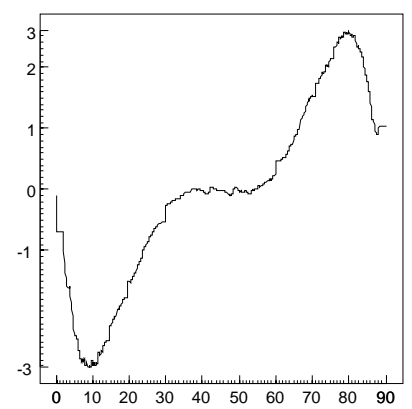

(d)

Fig. 6. Top: linear gray-level representation of $Q-Q^{\mathrm{T}}$ a) and $\tilde{Q}-\tilde{Q}^{\mathrm{T}}$ c), together with plots of the corresponding radial integrations - b) and d) - for the binary $\beta$ Del. Middle: $Q$ a) and $\tilde{Q} \mathbf{c}$ ), and the corresponding radial integrations- b) and d) -for the binary Moaï 1 . Bottom: the same as in Top for the binary $\gamma$ Per

It is interesting to note that the two versions of the method give, for these data, an equivalent result, even if the relevant ridge seems to be better defined using the reference star data.

\subsection{Moä̈ 1}

The close double star Moaï 1 (SAO 12917) was discovered during the observation from which the data used in this section are extracted. The approximate period evaluated by Carbillet et al. (1996c) is $\sim 13$ years. The separation vector $\boldsymbol{d}$ was deduced from this last paper using the cross-correlation technique (Aristidi et al. 1996). This corresponds to: $d=0^{\prime \prime} .11$ and $\mathrm{PA}=213^{\circ}$. The reference star observed was SAO 12929 from which we used 2617 frames, and 2619 for the binary.
Figure 6, second row, shows both the quantities $Q$ and $\tilde{Q}$, with their radial integrations $I_{Q}$ and $I_{\tilde{Q}}$. The value of $\theta$ derived from these quantities is: $\arctan \alpha=(42.0 \pm 2.0)^{\circ}$ or $\arctan \alpha^{\prime}=(41.5 \pm 3.5)^{\circ}$. The deduced value of $\alpha$ is then: $\alpha=0.900 \pm 0.065$ (or $\alpha^{\prime}=0.90 \pm 0.10$ ), that corresponds to a magnitude difference: $\Delta m=0.115 \pm 0.080$ (or $\Delta m^{\prime}=0.10 \pm 0.10$ ). This is in agreement with the value computed in the paper cited before and using both the cross-correlation technique and the fork algorithm (Bagnuolo 1988) $-\frac{1}{\alpha}=1.110 \pm 0.020$ and $\frac{1}{\alpha}=1.15 \pm 0.15$. The position angle is then confirmed to be: $\mathrm{PA}=213^{\circ}$ and the magnitude difference between the companion and the primary star in the red: $\Delta m_{R} \simeq 0.1$.

We can say that, here again, the method seems to take advantage of its use with the data of the reference star, the 
reference-less method giving anyway an acceptable value of the intensity ratio.

\section{3. $\gamma$ Per}

$\gamma$ Per is a giant eclipsing binary star of 17.8 years period whose orbit can be found in Hartkopf et al. (1996). The reference star observed was $\alpha$ Per from which we used 752 frames, and 443 for the binary. The separation and position angle computed from the visibility function were: $d=0^{\prime \prime} .20$ and $\mathrm{PA}=62^{\circ} / 242^{\circ}$.

Figure 6, third row, shows both the quantities $Q-Q^{\mathrm{T}}$ and $\tilde{Q}-\tilde{Q}^{\mathrm{T}}$, with their radial integrations $I_{Q-Q^{\mathrm{T}}}$ and $I_{\tilde{Q}-\tilde{Q}^{\mathrm{T}}}$. The value of $\theta$ derived from these quantities is: $\arctan \alpha=(79.5 \pm 1.0)^{\circ}$ - or $\arctan \alpha^{\prime}=(80.0 \pm 1.5)^{\circ}$. The deduced value of the intensity ratio is then: $\alpha=5.40 \pm 0.50$ (or $\alpha^{\prime}=5.70 \pm 0.90$ ), that corresponds to a magnitude difference: $\Delta m=-1.85 \pm 0.10$ (or $\Delta m^{\prime}=-1.90 \pm 0.15$ ). This first shows that the right position angle is $62^{\circ}$ and not $242^{\circ}$. Moreover, the absolute value of $\Delta m$ found is in agreement with the early speckle interferometric measurement of Labeyrie et al. (1974) that estimated a $\Delta m$ of 1-2 mag for a wavelength of $\lambda=6750 \AA$. More precisely, McAlister et al. (1982) estimated $\Delta m$ in the red to be at least greater than $1.4 \mathrm{mag}$, the estimated $\Delta m_{V}$.

\section{Discussion}

The method proposed in this paper to determine relative position and photometry of the components of a binary system consists of calculating ratios of PDFs: the twofold PDF of the double star speckle pattern computed for a space-lag $\rho$ equal to the star separation is divided by the twofold PDF of an unresolved star, computed for the same space-lag $\boldsymbol{\rho}$. Alternatively, and in a case of lack of a good reference star, the twofold PDF of the binary speckle pattern itself, computed for a space-lag $\boldsymbol{\rho}$ perpendicular to the star separation, may also be used as reference. The result, which is described in the text as the $Q$ (or $\tilde{Q}$ ) function, clearly evidences the region of the $\left(\Omega_{1}, \Omega_{2}\right)$ plane where $\Omega_{2}=\alpha \Omega_{1}$. This procedure, in some aspects, solves the problem of the PI technique emphasized in the introduction of this paper, i.e. the fact that it is a nonlinear approach for which there is no simple separation between functions of the object and of the speckle pattern. This pseudo-linear result was clearly illustrated in Fig. 1. Moreover, the use of a radial integration gives directly the value of $\alpha$ with no ambiguity on relative position of components.

Other representations may be considered to emphasize the dissymetry of the twofold PDF. For example, we have noticed that the ratio of $P$ to its transpose quantity $P^{\mathrm{T}}$ gave results similar to $Q-Q^{\mathrm{T}}$. For the sake of conciseness, these results are not reported here. In any case, since ratios are taken, a problem may arise when the twofold PDF used as a reference is equal to zero. This is not a major problem, but rather the effect of insufficient statistics in terms of number of samples. This problem could also be resolved if smoothed versions of twofold PDFs are used.

The ratio approach, even though we seek to obtain the linear relation discussed above, remains fully empirical. The question may arise about the meaning of these twofold PDFs ratios in terms of theory of probability and statistics. The ratio may be considered as the measure of some distance between probabilities, one bearing the information about the double star embedded in the speckle pattern, and the other being relevant to the PSF only. This approach is used in empirical hypothesis testing; however, the use of a ratio is not a common measure of distance (Allen 1990). Attempts were made to use differences of PDFs instead of ratios (Lyon 1993), but the results were found to be less attractive than the present ones.

Another possibility of a theoretical meaning for the ratio of PDFs is to refer to entropy and the information given by PDFs. The information associated with an event is equal to minus the logarithm of the probability of that event. The ratio we perform may be therefore linked to the difference between the information that comes from the law of probability of the intensity of a binary star speckle pattern, and that of an unresolved star. A deeper development of this approach, that we will not further develop here, would lead to the use of some Kullback-Leibler representation (Taupin 1988), of the form $P_{B} \log \left(P_{B} / P_{S}\right)$.

An alternative to the present method is to deal with CFs instead of PDFs. The division of the PDFs corresponds, in the Fourier space, to a deconvolution of the CFs. Surprisingly, we found that a division of the CFs leads to a similar result, since the $\mathrm{CF}$ computed for the binary (and for its separation) presents a characteristic ridge as well, which is also tremendously enhanced by dividing it by the CF of the PSF. This is an interesting behavior that we plan to study later.

The points discussed above are interesting problems of probability theory and signal processing, and will be developed elsewhere.

Several developments of the method are possible. A first one consists of the treatment of low-light level data. In this case, as discussed by Sultani et al. (1995), the PDF suffers a Poisson-Mandel transform that must be inverted. However, preliminary checks made on simulated data have shown that the information about $\alpha$ was already clearly visible in the ratio of low-light level PDFs. Another development is the extension of the procedure multiple stars. The analysis of triple stars speckle patterns is currently under processing and the results will be given in a near future.

\section{Conclusion}

We have developed in this paper a data processing method suitable for extracting astrometric information, absolute quadrant determination and relative photometry from 
speckle data of binary stars. The combination of classical visibility/autocorrelation calculus and our $Q$ function is proposed for this purpose. The results obtained for three binary stars (with different order of magnitude differences and angular separations) are very promising, and we are currently applying it as a routine analysis procedure for our speckle observations. The method being quite simple and fast, it could rapidly lead to near real-time processing.

Acknowledgements. The authors wish to thank J.C.Dainty for providing the WHT's data of $\gamma$ Per and for very helpful discussions about this paper; M. Carbillet thanks him more particularly for a five months stay in the Applied Optics group of Imperial College of London, under support of the European program Human Capital \& Mobility (contract ERB CHRX CT93 0335, coordinated by CEO Florence). Thanks are also due to J.-L.Prieur from Observatoire Midi-Pyrénées for the use of his specklegraph and his participation in the observations with the BLT. We are also indebted to B. Lopez and Y. Bresson for these observations, to H. Lantéri for stimulating and interesting discussions, and to C. Coulman for critical reading of the manuscript.

\section{References}

Aime C., 1987, J. Opt. (Paris) 18, 101-110

Aime C., 1993, Trends in Opt. Eng. 1, 15-34

Aime C., Ricort G., Perrier Ch., 1990, Exp. Astron. 1, 267-284

Aime C., Aristidi É., 1991, J. Opt. Soc. Am. A 8(9), 1434-1441

Aime C., Aristidi É., Lantéri H., Ricort G., 1993, Appl. Opt. $32,2747-2757$

Allen A.O., 1990, Probability, Statistics, and Queuing Theory, Second Edition, Academic Press

Aristidi É., Carbillet M., Lyon J.-F., Aime C., 1997a, (to be published in Astron. Astrophys.)

Aristidi É., Carbillet M., Prieur J.-L., et al., 1997b, (to be published in Astron. Astrophys.)

Bagnuolo W.G. Jr., 1988, Opt. Lett. 13 (10), 907-909
Bagnuolo W.G. Jr., Barry D.J., Mason B., Dombrowski E.G., 1990, "Results in speckle photometry" SPIE Proc. on: Amplitude and Intensity Spatial Interferometry 1237, Breckinridge J.B. (Ed.), 242-248

Bagnuolo W.G. Jr., Mason B.D., Barry D.J., Hartkopf W.I., McAlister H.A., 1992, AJ 103 (4), 1399-1407

Carbillet M., Ricort G., Aime C., Perrier Ch., 1996a, A\&A 310, 508-518

Carbillet M., Lopez B., Aristidi É., et al., 1996b, A\&A 314, $112-114$

Carbillet M., Aime C., Aristidi É., Ricort G., 1996c, in ESO Conference "Science with the VLT Interferometer", Garching-bei-München, Germany, 18-21 June 1996,

Coulman C.E., 1985, ARA\&A 23, 19-57

Couteau P., 1962, J. Observ. 45, 39

Cruzalèbes P., Tessier É., Lopez B., Eckart A., Tiphène D., 1996, A\&AS 116, 597-610

Hartkopf W.I., McAlister H.A., Franz O.G., 1989, AJ 98 (3), 1014-1039

Hartkopf W.I., Mason B.D., McAlister H.A., 1996, AJ 111 (1), 370-392

Knox T.K., Thompson B.J., 1974, ApJ 193, L45-L48

Labeyrie A., 1970, A\&A 6, 85-87

Labeyrie A., Bonneau D., Stachnik R.V., Gezari D.Y., 1974, ApJ 174, L147-L151

Lee Y.W., 1960, Statistical Theory of Communication, Wiley, New-York

Lyon J.-F., 1993, DEA report, Université de Nice-Sophia Antipolis, France

McAlister H.A., 1982, AJ 87 (3), 563-569

McAlister H.A., Hartkopf W.I., 1983, PASP 95, 778-781

Sultani F., Aime C., Lantéri H., 1995, Pure Appl. Opt. 4, 89103

Taupin D., 1988, Probabilities, data reduction, and error analysis in the physical sciences Editions de Sciences Physiques

Van Cittert P.H., 1931, Zeitschrift für Physics 69, 298

Weigelt G., 1977, Opt. Commun. 21, 55-59 